\title{
Distributions of simple RT with single and double stimuli
}

\author{
L. M. M. MEIJERS and E. G. J. EIJKMAN \\ Laboratory of Medical Physics and Biophysics, University of Nijmegen, Nijmegen, The Netherlands
}

\begin{abstract}
Two similar visual flashes spatially separated were used as stimuli in a simple RT experiment. The R'T distributions of the single stimuli were compared to cases with paired stimuli applied simultaneously (or nearly simultaneously). Spatial separation of simultaneous stimuli induces parallel processing. After comparing single-stimuli RT distributions with double-stimuli RT distributions, it was concluded that a race model with independent parallel processing provides the better fit to the data as compared to dependent parallel processing or partially parallel processing. Scanning models as well as signal summation models had to be rejected. The serial structure of the processing of single stimuli allows for a description of an exponential and a residual distribution. The analysis presented in this study is of importance for the concept of stages in serial processing of more complicated stimuli.
\end{abstract}

With visual signal processing, it is possible to induce parallel processing by using spatially separated stimuli. Because of the convergence of multiple inputs onto a single output, internal signals necessarily interact. The general notion is that with dualstimulus simple reactions, the same subprocesses are involved as with the single stimulus simple reactions for each separate stimulus. But with multiple-stimulus simple reactions, some of the subprocesses occur separately but in parallel, while others are in common. A number of interesting questions now arise: (1) How is subprocess interaction accomplished? (2) To what extent does parallel processing occur? (3) Do parallel processes act independently?

In thinking about subprocesses interaction, one can assume that the internal representation of activities caused by a temporally punctate stimulus gives rise to temporally punctate response, such as breaking a contact.

When the internal representation is temporally punctate, communication between subsystems may be regarded to occur with pulses. In the first part of the psychophysical system, pulses are transmitted along parallel channels-serial subsystems-each channel contributing a specific latency for the first pulse to arrive. Somewhere in the system, pulses will be guided into a common channel to emit a response through the motor system. An emitted response is therefore viewed as the outcome of a race between pulses transmitted through different channels. The first pulse to arrive at the common channel will emit a response; later parts of the signal will be without consequence. Following Kornblum (1973), such models will be referred to as "race models." The outcome of the race may be influenced by the experimenter through the time differences between stimuli within the multiple stimulus condition.

The general hypothesis of independent parallel processing has been proposed and supported in some studies. Kantowitz (1974) indicates that no sensory interaction is found among multiple stimuli. Lappin and Eriksen (1966) studied reaction time for effective inhibition of the response by a second stimulus. Ollman (1973) found that a model with stimulus and inhibition signal racing independently was basically right. Bernstein, Rose, \& Ashe (1970) reported RT studies on intersensory facilitation. Most of these reports implicitly assume that interaction, if present, occurs in an all or none manner.

On the other hand, information may be transmitted by temporally dispersed signals which evoke punctate responses when they cross a certain threshold. (Grice, 1968, 1972; John, 1967). These temporally dispersed signals may show summation phenomena in common channels, which still would be apparent if one of two signals was retarded.

Also, the question regarding the extent of parallel processing comes into play. It is already known that phototopical processing of visual stimuli extends deep into the cerebral structures. However, which circuits are involved in visuo-motor reactions remains unknown. It is certain that the very first visual information processing, at the retinal level, is performed in parallel.

Since the total latency distribution may be divided into two serial subsystem latency distributions, it is possible to construct models representing two different locations for the interaction. Special attention must be paid to the motor subsystem which is a common subsystem and must be included in the possible structures. Throughout most of the literature, motor time is assumed to be a constant delay, $t_{m}$. One would assume, therefore (Meijers \& Eijkman, 
1974) that it does not contribute to the variance of the total latency distribution. Many investigators have tried to decompose simple RT distributions in subsystem latency distributions (Christie \& Luce, 1956; Hohle, 1965, 1967; McGill \& Gibbon, 1965; Meijers, 1976; Snodgrass, 1969; Sternberg, 1969; Sternberg \& Knoll, 1973; Taylor, 1965). Normal distributions as well as other distributions have been proposed as possible subsystem latency distributions. Meijers (1976) indicated conditions and methods which allow a single-stimulus simple RT distribution to be broken down into an exponential subsystem latency distribution and a residual subsystem latency distribution.

Those latencies are assumed to be mutually independent. We have applied these techniques in a double-stimulation simple RT experiment to investigate possible relations between double-stimulus simple RT distributions and single-stimulus simple RT distributions.

The principal concern of the present study is the comparison of the RT distributions of simple RT in a two-channel paradigm. The two channels are stimulated well above threshold by different visual flashes sufficiently separated in the visual field for avoiding considerable lateral effects. This relatively simple paradigm, together with two identifiable serial subsystems, offers the opportunity to distinguish between partially and wholly separated channels.

In Figure 1, two possible structures are shown which are helpful in considering the case of a double stimulus. The motor subsystem latency density distribution is denoted by $m(t)=\delta(t-t)_{m}$, a Dirac delta function, producing a pulse at time $t=t_{m}$. The probability density function of an exponential subsystem latency is denoted by $l(t)$. The residual distribution $r(t)$ stands for the part of the latency not covered by $\mathrm{l}(\mathrm{t})$ or $\mathrm{m}(\mathrm{t})$.

The subsystems which receive the stimuli are
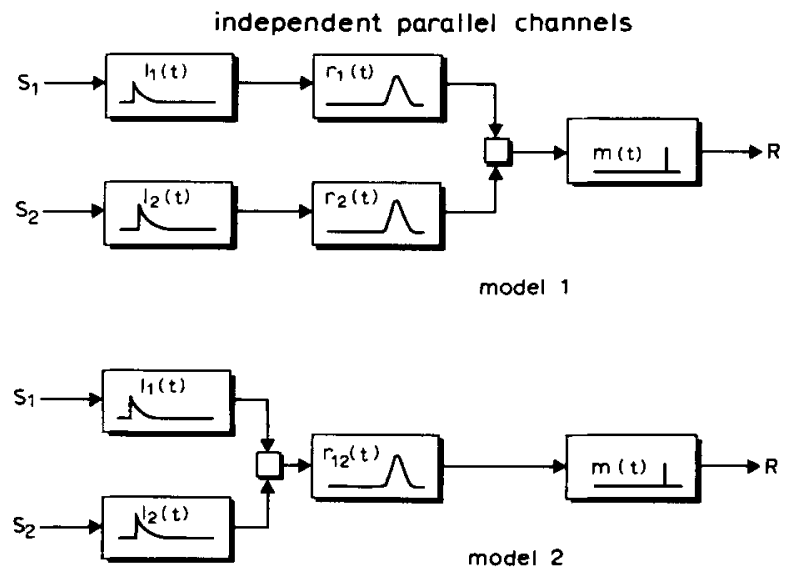

Figure 1. Structures of independent parallel channel models. For further explanation see text. assumed to be separate, in the sense that activity in one subsystem is not influenced by activity occurring in the other subsystem. The question now arises: are both subsystems, which are acting in parallel, stochastically independent? Correlation between subsystem properties might be caused, for example, by overall fluctuations in sensitivity or in attention. Since both subprocesses are unobservable, correlation between them cannot be determined in a straightforward manner. One way to solve this problem is to assume dependence or independence between parallel subsystems and to perform statistical analysis to support or reject these assumptions.

With simple reaction time experiments, it is very difficult to devise experiments which will answer the three questions selectively. It becomes necessary, therefore, to propose a model based upon several simultaneous assumptions and to test whether it will be rejected as a whole. Should the model fail, it will of course be difficult to decide which of its several assumptions might be at fault. If the model is not rejected, it will be still an insufficient proof of its validity since different structured sets of subsystems may give rise to RT distributions that have approximately the same validity with respect to the data at hand.

In the following sections, four different race models, a summation model, and a scanning model are proposed which incorporate assumptions regarding location of interaction and mutual dependence of parallel subsystems. For each model, the relations between the double-stimulus simple reaction time distribution (DSS-RT-distribution), denoted by $F_{12}(t)$, and the single-stimulus simple reaction time distributions (SSS-RT-distributions), denoted by $F_{1}(t)$ and $F_{2}(t)$, respectively, are derived. Each model will be tested with experimental RTs determined for single flashes, and for catch trials without a flash, each case occurring with equal probability.

\section{STRUCTURAL PROPOSALS}

\section{Model 1: Independent Parallel Channels}

For Model 1, a structure is proposed as depicted in Figure 1. A supplementary assumption is that operations of both parallel channels is stochastically independent. When operating in SSS-RT-trials the total latency distribution is expressed in terms of the component latency distribution, as shown below,

$$
\begin{array}{ll}
f_{j}(t)=l_{j}(t) * r_{j}(t) * m(t) & \\
F_{j}(t)=\int_{0}^{t} f_{j}(\tau) d \tau \quad j=1,2
\end{array}
$$

where ${ }^{*}$ denotes a convolution in time. By assumption, the observed response time, RT, in DSS-RTtrials, seen as the outcome of a race (Kornblum, 
1973) between both pulses in the parallel channels and added to the motor time latency $t_{m}$, is given by

$$
R T=\min \left(t_{c_{1}}, t_{c_{2}}\right)+t_{m},
$$

with $t_{c j}$ being the latency of the channel corresponding with stimulus $j$. It is assumed that $t_{c_{1}}$ and $t_{c_{2}}$ are independent, i.e.,

$$
P\left(R T-t_{m}>t\right)=P\left(t_{c_{1}}>t\right) \cdot P\left(t_{c_{2}}>t\right),
$$

or, expressed in the cumulative probability distribution functions, it becomes

$$
\mathrm{C}_{12}(\mathrm{t})=\mathrm{C}_{1}(\mathrm{t})+\mathrm{C}_{2}(\mathrm{t})-\mathrm{C}_{1}(\mathrm{t}) \cdot \mathrm{C}_{2}(\mathrm{t}) .
$$

Disregarding the constant motor time, $C_{1}(t)$ is equal to the SSS-RT-distribution $F_{1}(t)$, and similarly disregarding motor time, $t_{m}$, and the time delay $\Delta t$ between Stimulus 1 and Stimulus 2 during DSS-RTtrials, $C_{2}(t)$ is equal to $F_{2}(t)$. Equation 4 can therefore be used to predict the DSS-RT-distribution from the measured SSS-RT-distributions. Thus:

$$
F_{12}(t)=F_{1}(t)+F_{2}(t-\Delta t)-F_{1}(t) \cdot F_{2}(t-\Delta t)(5)
$$

Equation 5 permits comparison of predicted DSSRT-distribution $\hat{F}_{12}(t)$ on the basis of the measured $\dot{F}_{1}(t)$ and $\dot{F}_{2}(t)$ with the measured DSS-RTdistribution $\bar{F}_{12}(t)$.

\section{Model 2: Independent Partially Parallel Channels}

For Model 2, the structure is also shown in Figure 1. The expression for SSS-RT-distributions remains the same as for Model 1 expressed in Equation 1. In DSS-RT-trials, the race of both pulses along the parallel subsystems includes only the exponential subsystems. In this model, it is necessary to make a decision regarding the partitioning of the mean latency over the different subsystems.

At first sight, the effect of intensity upon the mean of the residual latency distribution results from the effect of intensity upon delays within all subprocesses, including the exponential subprocess. However, because stimulus intensity will have its most profound effect upon the first processing stages, it is obviously reasonable to attribute the difference in the means of the common residual latency distribution to differently delayed exponential subsystems. The means of both residual latency distributions found with the two single stimuli are consequently equalized. The residual latency distribution, $r_{12}(t)$, is defined as the arithmetic average of both residual latency distributions as estimated using the deconvolution method. The observed response time RT for this model is therefore given by

$$
R T=\min \left(t_{l_{1}}, t_{l_{2}}\right)+t_{r_{12}}+t_{m} .
$$

In this model $t_{l_{1}}$ and $t_{l_{2}}$ are the latencies of the respective exponential subsystems so that

$$
L_{12}(t)=L_{1}(t)+L_{2}(t)-L_{1}(t) \cdot L_{2}(t) .
$$

$L_{1}(t)$ equals the exponential subsystem latency distribution as estimated $\dot{F}_{1}(t)$ but delayed by the difference $\Delta \mu_{\mathrm{r}}^{1}$ between the means of both residual latency distributions as estimated from $\dot{F}_{1}(t)$ and $\dot{F}_{2}(t) \cdot L_{2}(t)$ equals the exponential subsystem latency distribution as estimated from $\tilde{F}_{2}(t)$ but delayed by the timer difference $\Delta t$ between Stimulus 1 and Stimulus 2 in DSS-RT-trials.

$$
\begin{array}{rlrl}
\mathrm{L}_{1}(\mathrm{t}) & =0 & \mathrm{t}<\Delta \mu_{\mathrm{r}}^{1} \\
& =\tilde{\lambda}_{1} \mathrm{e}^{-\tilde{\lambda}_{1}\left(\mathrm{t}-\Delta \mu_{\mathrm{r}}^{1}\right)} & \mathrm{t} \geqslant \Delta \mu_{\mathrm{r}}^{1}
\end{array}
$$

and

$$
\begin{array}{rlrl}
\mathbf{L}_{2}(t) & =0 & & t<\Delta t \\
& =\tilde{\lambda}_{2} e^{-\bar{\lambda}_{2}(t-\Delta t)} & t \geqslant \Delta t
\end{array}
$$

This DSS-RT-distribution may now be calculated according to

$$
\begin{aligned}
f_{12}(t) & =l_{12}(t) * r_{12}(t) \\
F_{12}(t) & =\int_{0}^{t}\left[l_{12}(\tau) * r_{12}(\tau)\right] d \tau,
\end{aligned}
$$

which permits comparison of predicted and measured DSS-RT-distributions.

\section{Model 3: Dependent Parallel Channels}

Instead of assuming independence between parallel subsystems, some kind of dependence may be possible. Because of the difficulties inherent in constructing a sensible model with partial dependence, i.e., a coefficient of correlation $|p|<1$, the extreme proposition of stochastically dependent parallel subsystems is assumed. The model depicted in Figure 1 is now equipped with a common factor which modulates the latencies of both channels simultaneously. Therefore, when $t_{c_{1}}$ is small, so is $t_{c_{2}}$. The observed response time, RT, is given by

$$
R T=\min \left(t_{c_{1}}, t_{c_{2}}\right)+t_{m} .
$$

Let $t_{e}$ denote the time for which $F_{1}(t)=F_{2}(t-\Delta t)$. As a consequence of the constant motor time, $t_{m}$, $C_{1}\left(t_{e}-t_{m}\right)=C_{2}\left(t_{e}-t_{m}-\Delta t\right)$. The assumption will be that $t_{c_{1}}=g\left(t_{c_{2}}\right)$ such that 


$$
\begin{array}{lll}
t_{c_{1}}<t_{c_{2}} & \text { for } & t_{c_{1}}<t_{e}-t_{m} \\
t_{c_{1}}=t_{c_{2}} & \text { for } & t_{c_{1}}=t_{e}-t_{m} \\
t_{c_{1}}>t_{c_{2}} & \text { for } & t_{c_{1}}>t_{e}-t_{m}
\end{array}
$$

Consequently,

$$
\begin{aligned}
P(R T<t) & =F_{1}(t) & t \leqslant t_{e} \\
& =F_{2}(t-\Delta t) & t>t_{e}
\end{aligned}
$$

So it is easy to see that

$$
\begin{array}{rlrl}
\hat{F}_{12}(t) & =\dot{F}_{1}(t) & & t \leqslant \tilde{t}_{e} \\
& =\dot{F}_{2}(t-\Delta t) & t>\tilde{t}_{e}
\end{array}
$$

Equation 13 permits comparison of $\hat{F}_{12}(t)$ and $\tilde{F}_{12}(t)$ for testing of this model.

\section{Model 4: Dependent Partially Parallel Channels}

The analogy between Model 1 and Model 3 is also present between Model 2 and Model 4. In Model 4, structured as is Model 2 in Figure 1, the stochastic dependence between the paralleled subsystems is substituted for stochastic independence. In discussing Model 2, remarks have been made regarding the properties of the subsystem latency distributions $l_{1}(t)$, $\mathrm{l}_{2}(\mathrm{t})$, and $\mathrm{r}_{12}(\mathrm{t})$. These remarks are equally true for the present model. The observed response time is:

$$
R T=\min \left(t_{l_{1}}, t_{l_{2}}\right)+t_{r_{12}}+t_{m} .
$$

Let $t_{e}$ be the time for which $L_{1}(t)=L_{2}(t)$, then by assumption (see Model 3)

$$
\begin{aligned}
P\left\{\min \left(t_{l_{1}}, t_{l_{2}}\right)<t\right\} & =L_{1}(t) \quad t \leqslant t_{e} \\
& =L_{2}(t) \quad t>t_{e}
\end{aligned}
$$

or

$$
\begin{aligned}
\mathrm{L}_{12}(\mathrm{t}) & =\mathrm{L}_{1}(\mathrm{t}) & \mathrm{t} \leqslant \mathrm{t}_{\mathrm{e}} \\
& =\mathrm{L}_{2}(\mathrm{t}) & \mathrm{t}>\mathrm{t}_{\mathrm{e}}
\end{aligned}
$$

Now the DSS-RT-distribution $F_{12}(t)$ may be predicted according to

$$
\begin{aligned}
f_{12}(t) & =l_{12}(t) * r_{12}(t) \\
F_{12}(t) & =\int_{0}^{1} l_{12}(\tau) * r_{12}(\tau) d \tau,
\end{aligned}
$$

permitting comparison of $\hat{\mathrm{F}}_{12}(\mathrm{t})$ and $\tilde{\mathrm{F}}_{12}(\mathrm{t})$ to test this model.

\section{Model 5: The Signal Summation Model}

When assuming a temporally dispersed signal representation of a stimulus during processing, the idea of an adjustable criterion applied to the response has received support from a number of studies of simple RT (Grice, 1968; John, 1967; Kohlfield, 1968; Murray, 1970). Here a decision mechanism is formulated in which the excitatory signal, $\mathrm{x}(\mathrm{t})$, of a stimulus is compared with a threshold value, $C$. When the excitatory strength, $x(t)$, exceeds $C$, a response is evoked. The threshold value or criterion is subject to a variety of influences and is assumed to be a random variable having a normal probability density function with mean $\langle\mathrm{C}\rangle$ and standard deviation $\sigma_{\mathfrak{c}}$. The admissibility of the normal probability density function stems from application of the central limit theorem. In dealing with latency data, it is assumed that the excitatory strength, $x(t)$, crosses threshold while $\mathrm{dx}(\mathrm{t}) / \mathrm{dt}>0$. The probability of a response at any given time will then be determined by the difference between average threshold value and excitatory strength, $x(t)$. From the probability $P(R T \leqslant t)$, the normal deviate $z(t)=[\langle C\rangle-x(t)] \sigma^{-1}$ is obtained. In these models, it is usually stressed that false alarms occur, which may be the result of a preparatory excitation, $x_{p}(t)$. So the excitatory strength, $x(t)$, is mostly treated as an addition of a preparatory signal, $x_{p}(t)$, and a sensory excitatory signal, $x_{j}(t)$ (no stimulus $j=0$, only one stimulus $\mathrm{j}=1$ or $\mathrm{j}=2$, a double stimulus $\mathrm{j}=1$ and $\mathrm{j}=2$ ):

$$
\mathrm{z}_{\mathrm{j}}(\mathrm{t})=\left\{\langle\mathrm{C}\rangle-\mathrm{x}_{\mathrm{j}}(\mathrm{t})-\mathrm{x}_{\mathrm{p}}(\mathrm{t})\right\} / \mathrm{o}
$$

If $\langle\mathrm{C}\rangle$ and $\sigma$ are assumed to be independent on the stimulus, the following set of $z$ functions may be obtained from experiment:

$$
\begin{aligned}
z_{0}(t) & =z_{p}(t) \\
z_{1}(t) & =z_{1}^{\prime}(t)+z_{p}(t) \\
z_{2}(t) & =z_{2}^{\prime}(t)+z_{p}(t) \\
z_{12}(t) & =z_{1}^{\prime}(t)+z_{2}^{\prime}(t)+z_{p}(t)
\end{aligned}
$$

The last three equations may be used to predict the first, which appears to be a rather powerful test to assess this model.

\section{Model 6: The Scanning Model}

The experiments are set up in such a way that the subject will not know whether a single or a double flash is to be expected for a stimulus. If scanning of possible stimulus sites is an important factor, the single stimuli would show an appreciable number of long RTs as compared with double-stimuli RTs. For 
instance, with scanning time of $50 \mathrm{msec}$, a variance of about $200(\mathrm{msec})^{2}$ would be added to the SSS-RTdistributions.

\section{METHODS}

Both stimuli were flashed squares of $3 \times 3 \mathrm{~cm}$, arranged in such a way as to minimize mutual interactions. The subjects faced a translucent screen of $7 \times 7 \mathrm{~cm}$ at a distance of $150 \mathrm{~cm}$. The flashed squares were situated in opposite corners of the screen. Background illumination was $0.1 \mathrm{~mL}$, which is in the mesopic range. The stimuli were flashed from behind onto the screen, with two flashtubes held in rectangular encasements. In the center of the translucent screen, a red-light-emitting diode was placed as a fixation point. The time course of the stimulus extended over a period of $2 \mathrm{msec}$. Coarse intensity control was achieved through selection of capacitors which discharged through the flashtubes. Fine control of intensity was done with a set of rotating Polaroid filters for each flashtube. The flashtubes were Xenon flashtubes (Metz type 502). Stimulus-integrated luminance ranged from $6 \mathrm{mLmsec}$ to $0.6 \mathrm{mLmsec}$.

The subject sat in a soundproof box where the stimulus display was visible through a window. In the box, an adjustable chinrest and foreheadrest were fitted to provide fixation of the head. The stimulus display was placed at eye level. The response key, a "morse key" which required an actuating force of $40 \mathrm{~g}$, was positioned on a table. The prestimulus warning signal was a click with a foreperiod of 800 msec. Subjects had to press the key as quickly as possible whenever they saw a flash. So no discrimination was required between the stimuli. The intertrial interval was $9 \mathrm{sec}$, invariabiy. The prestimulus signal was used to start two counters, one measuring time from prestimulus onset until stimulus onset, the other from prestimulus onset until response onset. This allows measurement of negative response times. The four stimulus configurations were: (1) no stimulus or catch stimulus, denoted by $S_{0}$; (2) the low-intensity Stimulus 1 , denoted by $S_{1} ;(3)$ the high-intensity Stimulus 2 , denoted by $S_{2}$; (4) Stimulus 1 followed by Stimulus 2, with a time interval adjustable between 0 and $50 \mathrm{msec}$ in steps of $1 \mathrm{msec}$ constituting the double stimulus, denoted by $S_{12}$.

The stimulus-response sequences were produced and processed by means of timers and counters interfaced with a programmable calculator. Stimuli were generated using a pseudorandom scheduling program. All relevant information was also stored on paper tape for processing, which exceeded the capabilities of the experiment-controlling computer.

Two male subjects, L.M. and W.R., 33 and 37 years old and highly practiced, participated in the experiments.

Following $5 \mathrm{~min}$ adaptation to the stimulus-display background, the subjects received 20 warm-up trials, and, after a short break of $20 \mathrm{sec}$, data collection was started over 100 trials. After five training sessions, every subject took part in 20 experimental sessions. Each of the four stimulus conditions was equally probable, and therefore the number of observations for each stimulus condition was limited to 25 per session in order to avoid effects of fatigue.

Following each session, statistics of RT distributions were presented for each stimulus condition. The logged tail distribution of $\mathrm{RT}$ vs. time were plotted on a X-Y recorder for each stimulus condition, facilitating visual inspection.

In cases of double stimuli, there generally was an interval between the two stimuli. This interval was constant for one session; it was introduced to assure a maximum overlap of the two RT distributions of both single stimuli and also in the case of different intensities of both stimuli.

Therefore, the stimulus with the highest intensity-yielding the shortest mean RT - was presented as the second of the pair. On the other hand, intensity difference induces unequal variances resulting in reduced overlap of distributions. Time delay and intensity difference between stimuli have to be balanced so as to yield distributions with maximum overlap. This balancing was accomplished by discarding experimental data with an overlap of less than $50 \%$. Moreover, latencies outside a range of $\left(\mu_{R T}-\right.$ $2 \sigma),\left(\mu_{\mathrm{RT}}+3 \sigma\right)$ were considered outliers. Outliers may have a profound effect upon the estimated parameters of the statistics; this is true for both the early responses released by accident and the very late responses which subjects often ascribe to inattention.

\section{RESULTS}

In Table 1, the experimental results of sessions are grouped for different interstimulus intervals.

It is not possible to come to a clear conclusion on the basis of measurements of each individual session. However, on the basis of chi-square values added over sessions, distinctions can be made. Because of repeated experiments, use will be made of the additivity property of the chi-square statistic, which states that the result of independent experiments considered together is equivalent to a $\chi_{s}^{2}$ value equal to the sum of the $\chi^{2}$ values of the experiments. The degrees of freedom, $v_{\mathrm{s}}$, are equal to the sum of the degrees of freedom of the experiments. For each experiment,

Table 1

Experimental Mean RT and Standard Deviations of Sessions With Different Interstimulus Intervals $\Delta t$

\begin{tabular}{|c|c|c|c|c|c|c|c|c|}
\hline Session & $\begin{array}{c}\Delta \mathrm{t} \\
\mathrm{msec}\end{array}$ & $\begin{array}{c}\overline{\mathrm{R}} \overline{\mathrm{T}}_{1} \\
\mathrm{msec}\end{array}$ & $\begin{array}{c}\mathrm{s}_{1} \\
\mathrm{msec}\end{array}$ & $\begin{array}{l}\overline{\mathrm{R}} \overline{\mathrm{T}}_{2} \\
\mathrm{msec}\end{array}$ & $\begin{array}{c}\mathbf{s}_{\mathbf{2}} \\
\mathrm{msec}\end{array}$ & $\begin{array}{l}\overline{\mathrm{RT}}_{12} \\
\mathrm{msec}\end{array}$ & $\begin{array}{c}\mathrm{s}_{12} \\
\mathrm{msec}\end{array}$ & FP* \\
\hline \multicolumn{9}{|c|}{ Subject L. M. } \\
\hline 01 & 1 & 176 & 12 & 175 & 10 & 171 & 8 & \\
\hline 02 & 1 & 190 & 10 & 186 & 9 & 181 & 6 & \\
\hline 03 & 10 & 182 & 20 & 180 & 12 & 183 & 12 & \\
\hline 04 & 11 & 193 & 20 & 180 & 10 & 192 & 13 & 1 \\
\hline 05 & 20 & 196 & 13 & 175 & 8 & 189 & 10 & \\
\hline 06 & 18 & 194 & 21 & 178 & 10 & 192 & 15 & 1 \\
\hline 07 & 23 & 176 & 8 & 169 & 7 & 176 & 9 & \\
\hline 08 & 30 & 190 & 30 & 173 & 12 & 189 & 13 & \\
\hline 09 & 5 & 178 & 12 & 172 & 10 & 175 & 9 & \\
\hline 10 & 3 & 171 & 14 & 160 & 7 & 160 & 11 & \\
\hline 11 & 3 & 197 & 16 & 189 & 12 & 182 & 11 & \\
\hline 12 & 10 & 194 & 19 & 181 & 11 & 190 & 12 & \\
\hline 13 & 12 & 184 & 16 & 175 & 10 & 182 & 10 & \\
\hline 14 & 4 & 180 & 13 & 173 & 12 & 176 & 10 & \\
\hline 15 & 5 & 196 & 15 & 192 & 13 & 188 & 10 & 1 \\
\hline 16 & 2 & 175 & 8 & 174 & 9 & 172 & 7 & 1 \\
\hline \multicolumn{9}{|c|}{ Subject W. R. } \\
\hline 01 & 2 & 170 & 12 & 165 & 10 & 163 & 8 & 1 \\
\hline 02 & 4 & 170 & 11 & 164 & 10 & 163 & 9 & 1 \\
\hline 03 & 10 & 181 & 13 & 170 & 10 & 176 & 9 & \\
\hline 04 & 11 & 182 & 14 & 169 & 11 & 175 & 12 & 1 \\
\hline 05 & 23 & 191 & 20 & 169 & 9 & 180 & 10 & \\
\hline 06 & 23 & 195 & 21 & 178 & 13 & 191 & 15 & \\
\hline 07 & 30 & 193 & 26 & 175 & 15 & 187 & 14 & \\
\hline 08 & 30 & 190 & 19 & 165 & 12 & 189 & 14 & \\
\hline 09 & 2 & 176 & 15 & 167 & 10 & 168 & 10 & \\
\hline 10 & 7 & 180 & 12 & 170 & 11 & 173 & 8 & 1 \\
\hline 11 & 4 & 172 & 10 & 171 & 11 & 172 & 9 & \\
\hline 12 & 9 & 182 & 14 & 171 & 11 & 178 & 10 & 1 \\
\hline 13 & 12 & 189 & 17 & 175 & 12 & 183 & 12 & \\
\hline 14 & 8 & 190 & 18 & 178 & 12 & 184 & 13 & \\
\hline 15 & 4 & 183 & 12 & 184 & 10 & 182 & 12 & 1 \\
\hline
\end{tabular}

${ }^{*} F P=$ number of false positives. 
the expected DSS-RT-distribution $\hat{\mathrm{F}}_{12}(\mathrm{t})$ was subdivided into four equal intervals for which expected and observed frequencies are compared and over which chi-square values are calculated. In Table 2, the chi-square values obtained are tabulated.

A comment must be made here regarding the use of the chi-square statistic. The chi-square statistic is a measure of the discrepancy between observed and theoretical frequencies. The theoretical frequencies are based upon probability rules applied to stochastic models. Population parameters of the model which have to be estimated from sample statistics affect the degrees of freedom. In our case, however, obtained and expected values are both stochastic variables, because $F_{1}(t)$ and $F_{2}(t)$ are used to calculate expected values. The chi-square test is therefore not fully applicable here. However, as far as we know, there are no tests available which are applicable to just this problem. We therefore decided to use the chi-square statistic while bearing in mind that the variations in these expected values would result in a larger chi-square value than that of a purely theoretically determined expectation.

A consequence of this is that the probability of rejection of a model will increase. A further consequence is that differentiation between models will be less decisive because the exact form of the chisquare statistic distributions is not known.

For subject W.R., the summed degrees of freedom amounted to 45 . Model 1 is clearly superior to Models 2, 3, and 4: $p<.001$ for Models 2, 3, and 4. For subject L.M., the summed degrees of freedom amounted to 48 . Model 1 is the most probable model: $\mathrm{p}<.01$ for Model 3 and $\mathrm{p}<.001$ for Models 3 and 4.

For Model 5, it is possible to predict the false alarm rate (reactions to $S_{0}$ ) from Expression 18. It appears that the measured false alarm rate is too low for all cases $(p<.01)$.

Finally, Model 6 predicts an appreciable increase of variance for single-stimulus cases. Differences in variance between single- and double-stimulus cases $\left(100 \mathrm{msec}^{2}\right)$ are too small to account for a much larger difference required by a scanning mechanism with a switching time of $10 \mathrm{~s}$ of milliseconds. Mechanisms with a switching time of $10 \mathrm{msec}$ or less cannot be ruled out, but this short interval is hardly feasible for a scanning mechanism of perceptual significance.

In order to display the comparison between predicted $\dot{F}_{12}(t)$ and measured $\dot{F}_{12}(t)$, consider that

$$
\frac{d}{d t}\left\{1 n\left[1-F_{12}(t)\right]\right\}=-\lambda_{12}(t),
$$

where $\lambda(t)$ denotes the time-dependent conditional probability density. The plot $1-\hat{\mathrm{F}}_{12}(\mathrm{t})$ vs. $1-$
Table 2

Summed Chi-Square Values of All Sessions

\begin{tabular}{cccccc}
\hline Subject & Model 1 & Model 2 & Model 3 & Model 4 & df \\
\hline W.R. & 40.1 & 85.5 & 81.2 & 166.3 & 45 \\
L.M. & 44.1 & 75.3 & 88.4 & 162.0 & 48 \\
\hline
\end{tabular}

$\check{\mathrm{F}}_{12}(\mathrm{t})$ on a $\log -\log$ scale has a number of interesting properties.

Property 1: When $\dot{\lambda}_{12}(\mathrm{t})$ equals $\hat{\lambda}_{12}(\mathrm{t})$ for every time point, the line with a slope of -1 originating from $(\mathrm{l}, \mathrm{l})$ is followed exactly. The minus sign is a consequence of the reversal of the horizontal axis, an effect which extends itself to Properties 2 and 3 also.

Property 2: The slope of the depicted function equals

$$
\begin{aligned}
-\frac{\mathrm{d}\left\{\ln \left[1-\dot{\mathrm{F}}_{12}(\mathrm{t})\right]\right\}}{\mathrm{d}\left\{\ln \left[1-\hat{\mathrm{F}}_{12}(\mathrm{t})\right]\right\}} & =-\frac{\frac{\mathrm{d}}{\mathrm{dt}}\left\{\ln \left[1-\dot{\mathrm{F}}_{12}(\mathrm{t})\right]\right\}}{\frac{\mathrm{d}}{\mathrm{dt}}\left\{\ln \left[1-\hat{\mathrm{F}}_{12}(\mathrm{t})\right]\right\}} \\
& =-\frac{\tilde{\lambda}_{12}(\mathrm{t})}{\hat{\lambda}_{12}(\mathrm{t})}
\end{aligned}
$$

Therefore, when the conditional probability densities are equal, the slope of the function will be -1 and run parallel to the diagonal line.

Property 3: When both distributions display exponential tail distributions, a straight line will appear with slope $-\tilde{\lambda}_{12}(t) / \hat{\lambda}_{12}(t)$.

In Figure 2, the data are displayed in this manner and represent the first eight experiments of each subject to give an impression of the variability of the results. The primary conclusion drawn from Figure 2 may be that the variability of the curves is considerable, but this is a consequence of the limited number of trials in an experiment.

In Model 1, the independent parallel channel model depicted in Figure 2, the diagonal line is followed, indicating conformity between predicted and measured DSS-RT-distributions.

In Model 2, the independent partially parallel channel model depicted in Figure 2, we can see that, with the exception of the initial segments, the curves run on the average in parallel to the diagonal line. This indicates equal conditional probability density for measured and expected DSS-RT-distribution. As a consequence of the time-boundedness of the residual latency distributions, the tail distributions of the expected DSS-RT-distributions for Models 1 and 2 are the same. Furthermore, we may conclude that the variability of the residual latency distribution is sufficient to distinguish between both models in a conclusive way.

Inspection of the plot of Model 3, the dependent parallel channel model in Figure 2, reveals that the 

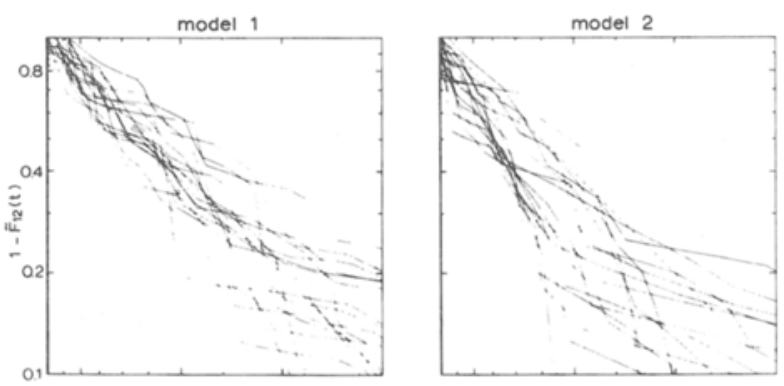

model 3
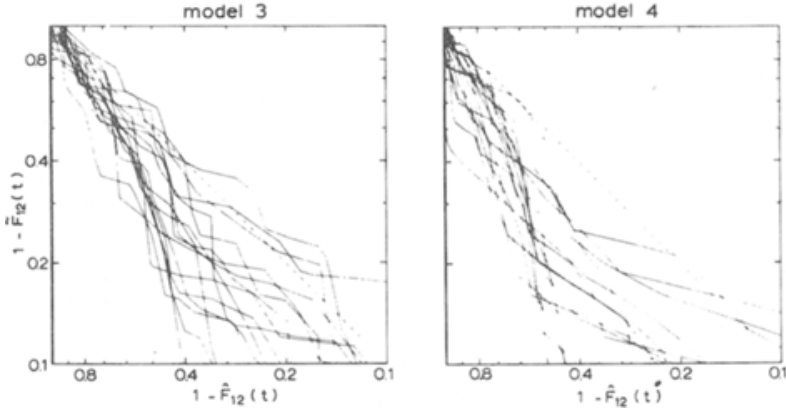

Figure 2. Graphical display of predicted DSS-RT-distribution $\dot{F}_{12}(t)$ vs. measured DSS-RT-distribution $\dot{F}_{12}(t)$ for each of the proposed race models.

predicted latency distribution lags the measured latency distribution considerably. The curves show that $\tilde{\lambda}_{12}(t) / \hat{\lambda}_{12}(t)>1$, indicating that predicted conditional density is less than measured conditional density at almost every time point. Comparison of Models 1 and 3 indicates that the independent operation of both channels favors the occurrence of short latencies.

In Model 4, the dependent partially parallel channel model depicted in Figure 2, the predicted latencies lag the measured latencies to an even greater extent and consequently the chi-square values indicate a worse fit. This also suggests that dependency and early interaction are both incorrect assumptions.

\section{DISCUSSION}

In present analysis shows the superiority of Model 1 over all five other models considered in this study. The question arises as to why all five other models, some of them closely related, were tested once the first model, and the simplest one, seemed acceptable. The comparison of six models, however, realizes a finer discrimination between the models of totally independent channels, of partially dependent channels, and of completely dependent channels. This is accomplished by the introduction of two identifiable subsystems, the one governed by delays with a simple exponential distribution and the other by delays with a residual distribution, the shape of which is determined by experiment. The fact that also with this subdivision of systems the completely independent process comes out first lends strong support to the race model for two channels, for which each can initiate the same motor system independently. Thus the motor system appears to contribute to measured RTs with a delay adding little variance to $\sigma^{2} \mathrm{RT}$, because an addition of variance would show as a dependency of the two channels.

The absence of interaction effects points to a fact of major significance. The only explanation for this experimental finding seems to be the assumption that the motor system involved in the response is initiated in an all or none manner by a sharply rising signal, such as a pulse or a step. This sharply rising signal when arriving first is the well-defined winner of the race. Had the trigger signal been dispersed in time, there would have been an overlap in arrival times of the two different trigger signals and more facilitating effects would have to be expected, with combined stimuli resulting in summation effects or dependency of channels during the period of overlap.

Pulse-shaped or step-like signals are not always accepted in descriptions of RT results. Counting models, for instance, are essentially summation models. In psychophysics of forward and backward masking, the smooth curves are very hard to explain with sudden onset of the signals carrying the necessary information.

On the other hand, the identification of processing stages advocated by Sternberg (1969) requires pulseshaped or step-like signals as stage initiators in order to obtain the necessary definition for stage duration. In models which relate simple RT with temporal order judgment, the steeply rising representation of stimuli is incorporated (see Gibbon \& Rutschmann, 1969, as well as Sternberg \& Knoll, 1973). In both papers, a model of the perception of temporal order is presented in which the difference of arrival time of the stimuli is the input signal for a center that judges temporal order. Both stimuli are supposed to be transmitted through independent channels towards the common center. The measurement of arrival time difference presupposes a temporally pulse-shaped representation of stimuli. Employing this model, Gibbon and Rutschmann (1969) were able to relate simple RT distributions to temporal order judgment for visual stimuli. Their findings also suggested that the motor component of reaction time adds little variance in comparison with that of the receptorsystem latency. With regard to the independence of channels and the motor time distributions with small variance, our findings are in agreement with theirs. On the other hand, in his thesis, Oostenburg (1975) argues that models for temporal order judgment probably operate upon amplitude of neural responses dispersed in time and evoked by the physical stimuli. It is entirely possible that the kind of experiment 
determines what shape of signals, pulse-like or dispersed, plays a part in the processing required for the response.

Finally one might be tempted to see a clear sign of temporally dispersed signals in recordings of EMGs also observed with movements required in knob pressings as used in many RT experiments. However, besides first arrival times, stochastic features of the EMG do not correlate with stimulus intensity or with the length of RT. One is forced to conclude that the motor system is initiated by an impulse if independent parallel channels operate or addition of moments (Sternberg, 1969) has been demonstrated. Then the fine structure of EMGs is completely determined by the motor system (Meijers \& Eijkman, 1974) and not by processing of sensory information.

\section{REFERENCES}

Bernstein, I. H., Rose, R., \& Ashe, V. M. Energy integration in intersensory facilitation. Journal of Experimental Psychology, 1970, 86, 196-203.

Christie, L. S., \& Luce, R. D. Decision structure and time relations in simple choice behaviour. Bulletin of Mathematical Biophysics, 1956, 8, 89-112.

Gibbon, J., \& Rutschman, R. Temporal order judgement and reaction time. Science, 1969, 165, 413-415.

Grice, G. R. Stimulus intensity and response evocation. Psychological Review, 1968, 75, 359-373.

GRICE, G. R. Application of a variable criterion model to auditory reaction time as a function of the type of catch trial. Perception \& Psychophysics, 1972, 12, 103-107.

НонцE, R. H. Inferred components of reaction times as functions of fore-period duration. Journal of Experimental Psychology, $1965,69,382-386$.

Hohle. R. H. Component process latencies in reaction times of children and adults. In L. P. Lipsett \& C. C. Spiker (Eds.), Advances in child development and behavior (Vol. 3). New York: Academic Press, 1976. Pp. 225-261.

JokN, I. D. A statistical decision theory of simple reaction time. Australian Journal of Psychology, 1967, 19, 27-34.
Kantowitz, B. H. Ch. II. In B. H. Kantowitz (Ed.), Human in. formation processing, tutorials in performance and cognition. Hillsdale, N.J: Erlbaum, 1974.

KOHLFELD, D. L. Stimulus intensity and adaptation level as determinants of simple reaction time. Journal of Experimental Psychology, 1968, 76, 468-473.

Kornblum, S. Simple reaction time as a race between signal detection and time estimation: A paradigm and model. Perception \& Psychophysics, 1973, 13, 108-112.

LAPPIN, J. S., \& Eriksen, C. W. Use of delayed signal to stop a visual reaction time response. Joumal of Experimental Psychology, 1966, 72, 805-811.

McGill, W. J., \& GibBon, J. The general gamma distribution and reaction time. Journal of Mathematical Psychology, 1965, 2, $1-18$.

MeIJERS, L. M. M. Human simple reaction time and psychophysical system analysis. Thesis, University of Nijmegen, The Netherlands, 1976.

MeiJers, L. M. M., \& Eujkman, E. G. J. The motor system in simple reaction time experiments. Acta Psychologica, 1974, 38, 367-377.

MURRAY, G. H. Stimulus intensity and reaction time: Evaluation of a decision theory model. Journal of Experimental Psychology, 1970, 84, 383-391.

Oostenbrug, M. W. M. Temporal acuity in foveal vision. Thesis, University of Groningen, The Netherlands, 1975.

Ollman, R. T. Simple reactions with random countermanding of the "Go" signal. In S. Kornblum (Ed.), Attention and performance IV. New York: Academic Press, 1973. Pp. 571-581.

SNoDgrass, J. Foreperiod effects in simple RT: Anticipation or expectancy. Journal of Experimental Psychology Monograph, $1969,79,1-19$.

STERNBERG, S. The discovery of processing stages: Extension of Donders' method. In W. G. Koster (Ed.), Attention and performance II. Amsterdam: North Holland, 1969. Pp. 276-315.

SternB ERG, S., \& KNOLL, R. L. The perception of temporal order: Fundamental issues and a general model. In S. Kornblum (Ed.), Attention and performance IV. New York: Academic Press, 1973. Pp. 629-685.

TAYLoR, D. H. Latency models for RT-distributions. Psychometrica, 1965, 30, 157-163.

(Received for publication August 2, 1976; revision accepted March 29, 1977.) 